14 Dempsey JA, Xie A, Patz DS, et al. Physiology in medicine: obstructive sleep apnea pathogenesis and treatment considerations beyond airway anatomy. J Appl Physiol (1985) 2014; 116: 3-12.

15 White DP. Pathogenesis of obstructive and central sleep apnea. Am J Respir Crit Care Med 2005; 172: $1363-1370$.

16 Okie S. A flood of opioids, a rising tide of deaths. N Engl J Med 2010; 363: 1981-1985.

\title{
Linezolid tolerability in multidrug-resistant tuberculosis: a retrospective study
}

\author{
To the Editor:
}

Linezolid is used off-label to treat multidrug-resistant (MDR) and extensively drug-resistant (XDR) tuberculosis (TB) $[1,2]$. Recently, two systematic reviews and meta-analyses pointed out its promising efficacy $[3,4]$. However, linezolid toxicity may outweigh its potential benefits. Indeed, adverse events were notified in almost $60 \%$ of the treated cases, with a high incidence of severe events such as anaemia, peripheral neuropathy, optic neuritis, and thrombocytopenia. Decreased linezolid doses were associated with significantly lowered toxicity [5]. Furthermore, therapeutic drug monitoring (TDM) has increasingly been recognised as an asset in the field of TB treatment [6, 7]. TDM may assess individual linezolid exposure, especially since the drug shows a large inter-individual variability [8] and important pharmacological interactions were observed [6]. However, there is no clear association between linezolid exposure and adverse events. Drug exposure is not routinely evaluated, either in prospective studies or in routine care. Unfortunately, TDM is not routinely incorporated into the study designs of research upon MDR-TB and linezolid [9, 10]. Therefore, we aimed to retrospectively investigate linezolid safety and tolerability in relation to linezolid exposure in MDR-TB patients.

A retrospective study was conducted at two TB reference hospitals, the Tuberculosis Center Beatrixoord (University Medical Center Groningen, Haren, the Netherlands) and the Tuberculosis Reference Center for MDR-TB and HIV-TB E. Morelli Hospital (Sondalo, Italy). We selected patients that received linezolid as a part of their TB-treatment regimen [11]. Retrospective, consecutive data were retrieved by two researchers and consisted of: patient demographic, clinical, and epidemiological characteristics; drug susceptibility testing (DST) results; linezolid serum concentrations, reflected by an area under the time-concentration curve from 0-24 h (AUC0-24); and detailed information on the disease and treatment. If AUCs were available for different linezolid doses, the curve of the dose that was administered during the longest period of time was used for analysis. To assess safety and tolerability, data on adverse events, e.g. leukopenia, peripheral neuropathy, anaemia, thrombocytopenia, optic neuritis and gastrointestinal disorders, were collected from the hospital records or laboratory files using local reference values and procedures, representing clinical practice.

Of the original 112 patients 54 were excluded because they were: aged $<18$ years $(n=7)$, lacked data due to recent admission $(n=9)$, had drug-susceptible TB $(n=4)$, or their TDM data were unavailable $(n=34)$. Patients aged $<18$ years were excluded to rule out age-related developmental changes that might influence pharmacology. DST revealed susceptibility and resistance to a median (interquartile range (IQR)) number of $6.0(4.0-8.0)$ and $8.0(7.0-10.0)$ drugs) respectively. The median (IQR) for the number of different active drugs administered at any one time-point during treatment was 6.0 (5.0-7.0). DST revealed a median (IQR) minimum inhibitory concentration for linezolid of $0.5 \mathrm{mg} \cdot \mathrm{L}^{-1}\left(0.25-0.5 \mathrm{mg} \cdot \mathrm{L}^{-1}\right)$.

Table 1 describes the linezolid AUC0-24, cumulative dose, and number of days of exposure to linezolid for patients with and without linezolid-related adverse events. The distribution of AUC0-24 was the same for patients with and without anaemia $(\mathrm{p}=0.299)$, leukopenia $(\mathrm{p}=0.314)$, optical neuritis $(\mathrm{p}=0.612)$, and peripheral neuropathy $(\mathrm{p}=0.261)$. The distribution of minimum serum concentration ( $\mathrm{Cmin})$ of linezolid was the same across the categories anaemia $(p=0.169)$, leukopenia $(p=0.207)$, optical neuritis $(p=0.98)$, and peripheral neuropathy $(\mathrm{p}=0.477)$. Peripheral neuropathy was observed in $11(19 \%)$ patients. The distribution of linezolid cumulative dosage in $\mathrm{mg} \cdot \mathrm{kg}^{-1}(\mathrm{p}=0.041)$ and number of days of exposure to linezolid ( $\mathrm{p}=0.003$ ) were significantly higher in the patient group with peripheral neuropathy. Five $(9 \%)$ out of 45 patients (13 patients had missing data) had leukopenia: no differences were identified in cumulative linezolid dose or in days of exposure to linezolid ( $\mathrm{p}=0.194$ and 0.065 , respectively). Only one patient had optical neuropathy. 
TABLE 1 Linezolid AUC0-24, cumulative dose, and days of exposure to linezolid for patients with and without linezolid-related adverse events

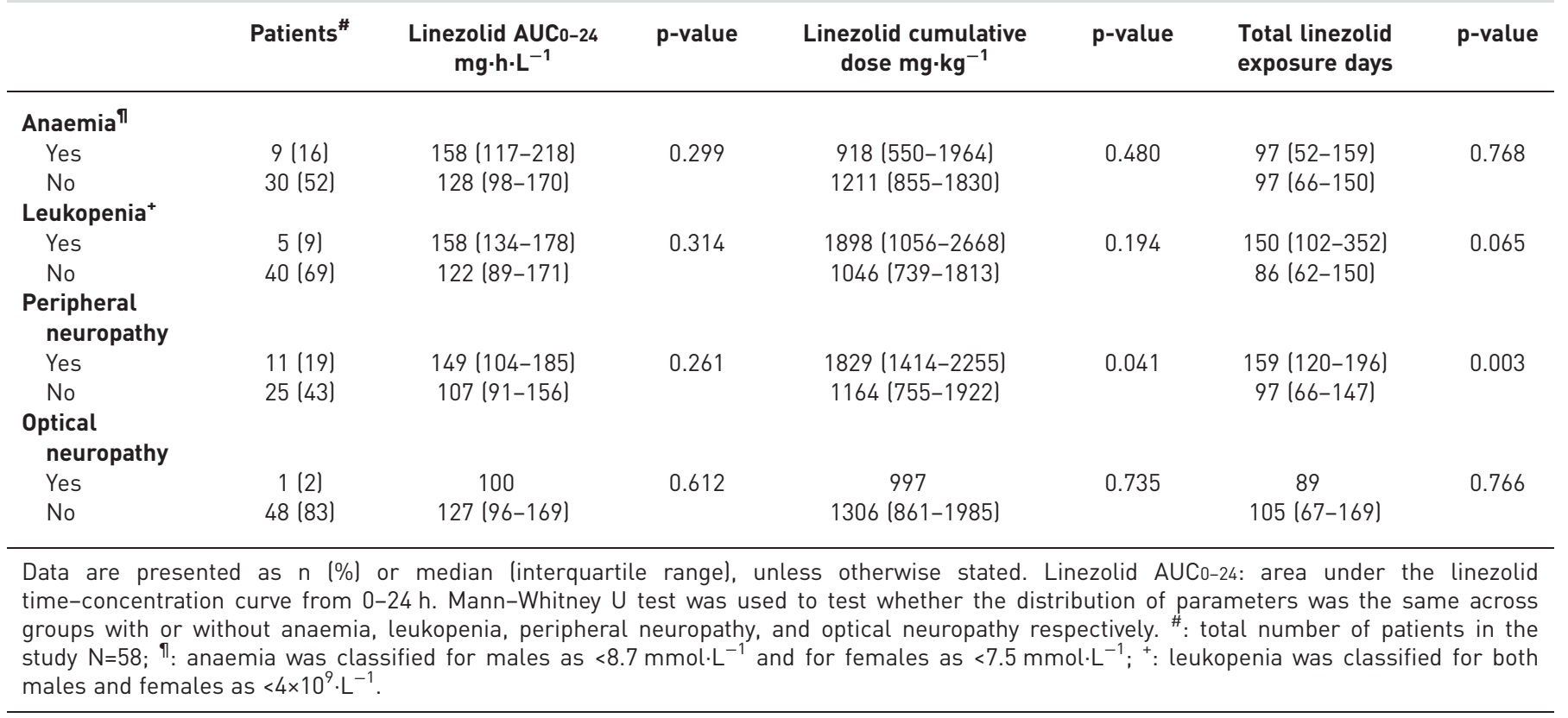

In this study we aimed to investigate linezolid toxicity in relation to linezolid exposure. The proportion of adverse events in the retrospective cohort was lower than previously reported in a recent large meta-analysis [4]. In our cohort we found that anaemia, peripheral neuropathy and optical neuritis occurred in 16\%, 19\%, and $2 \%$ of the patients, respectively. Whereas, in the meta-analysis [4], it was found that anaemia, peripheral neuropathy, and optic neuritis occurred in $38 \%, 47 \%$, and $13 \%$ of the patients, respectively. Comparison of dose-related effects is difficult due to the multitude of the included studies in the meta-analysis, the non-fixed dose in our retrospective study, and the small overlap of data [4]. The fact that in Tuberculosis Center Beatrixoord, one of the TB reference hospitals studied, $75 \%$ of the patients included in the study received erythropoietin in a dose of $2000 \mathrm{IU}$ twice a week, in an attempt to prevent anaemia, further complicates comparisons. This might, in part, explain the lower occurrence of anaemia in our cohort compared with that of the meta-analysis [10]. However, retrospective analysis of the haemoglobin levels revealed no significant differences between patients who did or did not receive a preventive erythropoietin.

The cumulative linezolid dosage and the number of days patients were exposed to linezolid were observed to be statistically significantly higher in patients who experienced peripheral neuropathy. A possible explanation that the cumulative linezolid dosage and days of exposure to linezolid, in contrast to linezolid Cmin or AUC, are associated with peripheral neuropathy might be that even the lower linezolid concentrations are above the mitochondrial toxicity threshold [12].

Several methodological study limitations can be identified, in particular the limited sample size, the retrospective epidemiological nature of our study, and missing data. Unfortunately, a large number of patients had to be excluded. Despite the fact that the inclusion and exclusion process has been transparently documented, a selection bias cannot be ruled out. Furthermore, linezolid exposure data were cross-sectionally computed. During the course of treatment the linezolid dosages or clinical parameters might also have changed. Due to the above factors it is difficult to attribute adverse effects solely to linezolid. Furthermore, differences between the two study settings are also a limitation. Data were collected at two hospitals by two different researchers. We cannot exclude general differences in standard of care, treatment, and monitoring. However, the impact of any observed differences in treatment regimens is unclear since all regimens are designed based on WHO guidelines [11]. Despite the retrospective nature of this study and the above mentioned limitations, our study supports the increasingly important position of linezolid in MDR-TB treatment regimens. Toxicity is frequent with higher doses, i.e. $600 \mathrm{mg}$ twice daily [8]. However, with reduced dosage, adverse events appear manageable and infrequent. Our findings justify confirmation by prospective cohort data allowing to further study linezolid exposure in relation to adverse events in MDR-TB patients [13]. This will become even more important when the patent of linezolid expires in the following years. The expected drop in costs will allow a larger population of patients to be 
treated with this drug. International collaboration in MDR/XDR-TB research will probably become critically important to facilitate the merging of data to provide large cohort sizes that can be used to explore important clinical questions, such as: what is the role of linezolid drug exposure in the treatment of MDR-TB? Perhaps linezolid dried blood spot [14] or a limited sampling strategy [15] could facilitate TDM in such studies to assess linezolid drug exposure.

In conclusion, our retrospective study suggests that the treatment regimens containing linezolid were well tolerated, with relatively low frequency of adverse events. No associations were observed between linezolid AUC0-24 and adverse events. However, peripheral neuropathy seemed to be mediated by cumulative dose and days of exposure to linezolid.

0 @ERSpublications

Linezolid for MDR-TB is well tolerated but peripheral neuropathy is related to cumulative dose and days of exposure http://ow.ly/Nx8wC

Mathieu S. Bolhuis $^{1}$, Simon Tiberi ${ }^{2}$, Giovanni Sotgiu ${ }^{3}$, Saverio De Lorenzo ${ }^{4}$, Jos G.W. Kosterink ${ }^{1,5}$, Tjip S. van der Werf ${ }^{6,7}$, Giovanni Battista Migliori ${ }^{8}$ and Jan-Willem C. Alffenaar ${ }^{1}$

${ }^{1}$ Dept of Clinical Pharmacy and Pharmacology, University Medical Center Groningen, University of Groningen, Groningen, The Netherlands. ${ }^{2}$ Dept of Infection, Barts Healthcare NHS Trust, London, UK. ${ }^{3}$ Clinical Epidemiology and Medical Statistics Unit, Dept of Biomedical Sciences, University of Sassari, Research, Medical Education and Professional Development Unit, AOU Sassari, Italy. ${ }^{4}$ E. Morelli Hospital AOVV, Reference Center for MDR-TB and HIV-TB, Sondalo, Italy. ${ }^{5}$ Dept of Pharmacy, Pharmacotherapy and Pharmaceutical Care, University of Groningen, Groningen, The Netherlands. ${ }^{6}$ University of Groningen, University Medical Center Groningen, Dept of Internal Medicine, Groningen, The Netherlands. ${ }^{7}$ University of Groningen, University Medical Center Groningen, Dept of Pulmonary Disease and Tuberculosis, Groningen, The Netherlands. ${ }^{8}$ WHO Collaborating Centre for Tuberculosis and Lung Diseases, Fondazione S Maugeri, Care and Research Institute, Tradate, Italy.

Correspondence: Mathieu S. Bolhuis, University Medical Center Groningen, Department of Clinical Pharmacy and Pharmacology attn. Hanzeplein 1, PO box 30 001, 9700 RB, Groningen, The Netherlands. E-mail: m.s.bolhuis@umcg.nl

Received: April 172015 | Accepted after revision: May 072015 | First published online: July 092015

Conflict of interest: Disclosures can be found alongside the online version of this article at erj.ersjournals.com

\section{References}

1 Lee M, Lee J, Carroll MW, et al. Linezolid for Treatment of Chronic Extensively Drug-Resistant Tuberculosis. N Engl J Med 2012; 367: 1508-1518.

2 Tang S, Yao L, Hao X, et al. Efficacy, safety and tolerability of linezolid for the treatment of XDR-TB: a study in China. Eur Respir J 2015; 45: 161-170.

3 Cox H, Ford N. Linezolid for the treatment of complicated drug-resistant tuberculosis: a systematic review and meta-analysis. Int J Tuberc Lung Dis 2012; 16: 447-454.

4 Sotgiu G, Centis R, D'Ambrosio L, et al. Efficacy, safety and tolerability of linezolid containing regimens in treating MDR-TB and XDR-TB: systematic review and meta-analysis. Eur Respir J 2012: 40; 1430-1442.

5 De Lorenzo S, Alffenaar JW, Sotgiu G, et al. Efficacy and safety of meropenem-clavunate added to linezolid-containing regimens in the treatment of M/XDR-TB. Eur Respir J 2013; 41: 1386-1392.

6 Bolhuis MS, van Altena R, van Soolingen D, et al. Clarithromycin increases linezolid exposure in multidrug-resistant tuberculosis patients. Eur Respir J 2013; 42: 1614-1621.

7 Srivastava S, Peloquin CA, Sotgiu G, et al. Therapeutic drug management: is it the future of multidrug-resistant tuberculosis treatment? Eur Respir J 2013; 42: 1449-1453.

8 Alffenaar JW, van Altena R, Harmelink IM, et al. Comparison of the pharmacokinetics of two dosage regimens of linezolid in multidrug-resistant and extensively drug-resistant tuberculosis patients. Clin Pharmacokinet 2010; 49: 559-565.

9 Singla R, Caminero JA, Jaiswal A, et al. Linezolid: an effective, safe and cheap drug for patients failing multidrug-resistant tuberculosis treatment in India. Eur Respir J 2012; 39: 956-962.

10 Bolhuis MS, Pranger AD, Alffenaar JW. Linezolid: safety and efficacy monitoring. Eur Respir J 2012; 39: 1275-1276.

11 Falzon D, Jaramillo E, Schünemann HJ, et al. WHO guidelines for the programmatic management of drug-resistant tuberculosis: 2011 update. Eur Respir J 2011; 38: 516-528.

12 De Vriese AS, Coster RV, Smet J, et al. Linezolid-induced inhibition of mitochondrial protein synthesis. Clin Infect Dis 2006; 42: 1111-1117.

13 Sotgiu G, Pontali E, Migliori GB. Linezolid to treat MDR-/XDR-tuberculosis: available evidence and future scenarios. Eur Respir J 2015; 45: 25-29.

14 Vu DH, Bolhuis MS, Koster RA, et al. Dried blood spot analysis for therapeutic drug monitoring of linezolid in patients with multidrug-resistant tuberculosis. Antimicrob Agents Chemother 2012; 56: 5758-5763.

15 Alffenaar JW, Kosterink JG, van Altena R, et al. Limited sampling strategies for therapeutic drug monitoring of linezolid in patients with multidrug-resistant tuberculosis. Ther Drug Monit 2010; 32: 97-101. 